\title{
MICROMECHANICS OF FERROIC FUNCTIONAL MATERIALS
}

\author{
John E. Huber \\ Department of Engineering Science \\ University of Oxford \\ Parks Rd., Oxford, OX1 3PJ, UK. \\ John.Huber@eng.ox.ac.uk
}

\begin{abstract}
Summary
This chapter introduces a range of ferroic functional materials including ferroelectrics, ferroelastics and ferromagnets. Coupling among the different types of ferroic order results in multiferroic behavior that is of importance in transducers and memory devices. The physical laws governing each type of material are compared, noting the closely analogous governing equations. However, attention is also given to the differences in behaviour that can necessitate distinct modeling approaches. The general form of Eshelby tensor for coupled ferroics is introduced and methods for estimating the properties of composites, including self-consistent and Mori-Tanaka schemes, are briefly described. The chapter then focuses on the analysis of a commonly encountered arrangement of microstructure: a composite laminate comprising distinct crystal variants of the same physical phase. Rules are presented for determining how laminates can form with a minimum energy arrangement of layers, and methods are described for estimating the resulting material properties of the composite. An application to the microstructure of freestanding polycrystalline thin films is described.
\end{abstract}

Keywords: Ferroic materials, ferroelectric, ferroelastic, ferromagnetic, magnetic shape memory alloy, laminate, polycrystal. 


\section{Introduction}

Ferroics are materials that exhibit any one or more of the properties ferroelectricity, ferroelasticity, or ferromagnetism (Aizu, 1970; Schmid, 1994). They are of importance in the development of smart material systems and functional devices. Included in this class of materials are ferroelectrics that posses a remanent polarization, which can be reversed by the application of electric field. Also included are the ferroelastic materials that possess distinct, symmetryrelated crystal variants with differing states of stress-free strain. These materials can be deformed by converting one crystal variant into another and the process may be reversed allowing cyclic straining. The resulting effect is named ferroelasticity by analogy with ferroelectricity and ferromagnetism. Ferromagnetic materials possess a remanent magnetization that can be reversed by the application of magnetic field. Each of the aforementioned material types has the capacity to be ordered in a way that stores a permanent state - a polarization, strain or magnetization and for that state to be altered by the application of external fields. This leads to applications in switching and memory devices. Ferroic order is sometimes taken to include other orderings, such as ferrimagnetism or ferrielectricity wherein the presence of positive and negative magnetization or polarization produces a partial balance, and antiferromagnetism or antiferrolectricity where there is complete balance eliminating the macroscopic magnetization or polarization. Ferrotoroidicity, the possession of a circulation of magnetization, has been ascribed as a further form of ferroic order (Van Aken et al., 2007). However, as this ordering is relatively rare, the present chapter will focus on the three main types of ferroic order and relations between them.

Where more than one ferroic order is present, and especially where there is coupling between ferroic orders, a material may be called multiferroic. The term is sometimes reserved for materials exhibiting both ferroelectricity and ferromagnetism, coupled through the magnetoelectric effect, such that electric fields can induce magnetization and magnetic fields can induce polarization. However, the broader definition involves the pairing of any two or more ferroic orders. Using this broader definition of multiferroicity, most ferroelectrics are multiferroic since they typical display both ferroelectricity and ferroelasticity. Similarly, Magnetic shape memory alloys display both conventional ferromagnetic behavior and ferroelasticity in the form of shape memory. These materials are intrinsically multiferroic: multiple ferroic orders are present in a single phase. However, strong coupling between 
ferroelectricity and ferromagnetism in a single phase is relatively rare (Eerenstein et al., 2007). Instead, composites of ferroelectric and ferromagnetic materials can achieve strong magnetoelectric coupling mediated by continuity of displacement at the interface between phases (Nan et al., 2008). Effectively, application of a magnetic field can cause magnetization and strain in the ferromagnetic phase, and the coupling of strain into the ferroelectric phase can modify polarization. This composite route to strong magnetoelectric coupling is important because of the potential applications in memory devices where combinations of electrical and magnetic read-write operations may be desirable.

Returning to the main ferroic orders, their interrelations are shown in Fig. 1. Commonly, the presence of a ferroic order implies symmetry that allows useful coupled effects. Hence ferroelectrics are typically piezoelectric (though not all piezoelectrics are ferroelectric) and so forth. The strong couplings give rise to applications where transduction between electrical magnetic and mechanical energy occurs. Then the primary role of the material is not structural and these materials are referred to as functional. The present chapter deals with ferroic functional materials, which display ferroic order and coupling that may be used for applications such as sensing, actuation, transduction, memory devices and others. In each case, the ferroic order results from a symmetry breaking phase transition, normally a high temperature symmetric phase with no ferroic order transforming upon cooling into a low symmetry phase with ferroic order. In both ferroelectrics and ferromagnetics the transition temperature is called the Curie Temperature, $T_{\mathrm{C}}$. The corresponding transition in shape memory alloys is an austenite-martensite transformation, producing a low temperature martensite phase with several symmetry related crystal variants. 


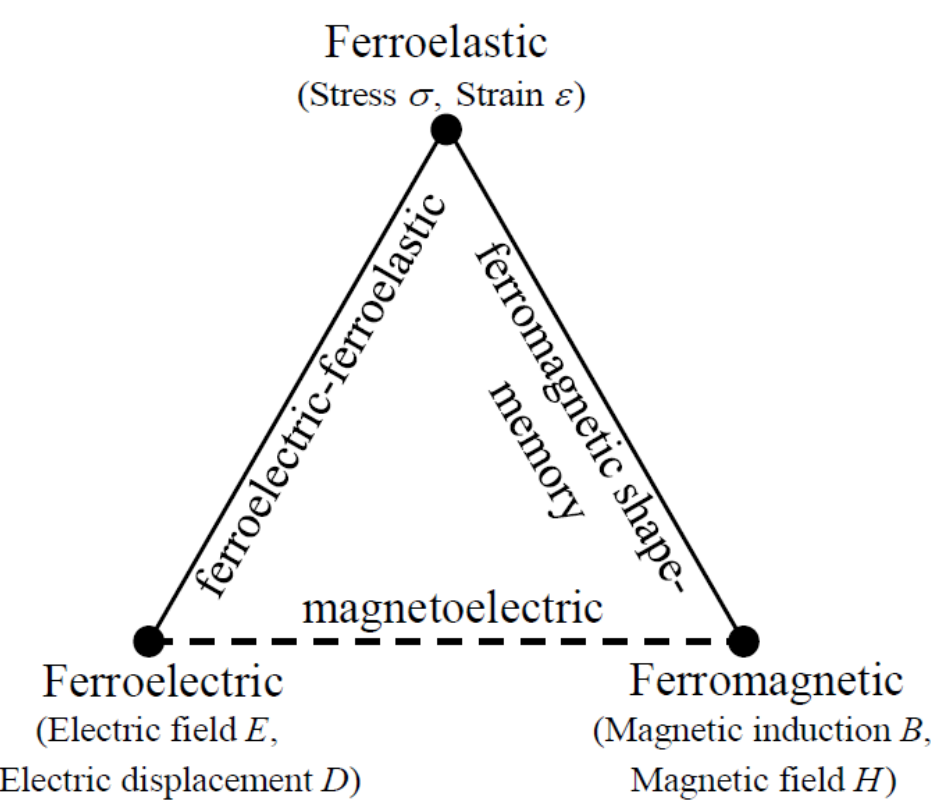

Fig. 1. The main ferroic orders and relations among them.

As well as introducing the three main ferroic orders, Fig. 1 also introduces the field variables associated with each type of ferroic material at uniform and constant temperature. In ferroelasticity the key variables are mechanical stress $\sigma$ and strain $\varepsilon$; ferroic ordering allows for a remanent strain $\varepsilon^{\mathrm{R}}$ to be present. The corresponding variables in ferroelectricity are electric field $E$ and electric displacement $D$ with remanent polarization $P^{\mathrm{R}}$, being an electric displacement at zero electric field, due to ordering. In ferromagnets, magnetic induction $B$ (also called magnetic flux) and magnetic field $H$ are the field variables, with a remanent magnetization $M$ describing the state when applied fields are absent.

\section{Governing equations, constitutive equations and material description}

Let us first consider the physical laws that govern ferroic materials, and the specific forms of these laws relevant to quasi-static conditions. Mechanical equilibrium and Gauss' law for electric and magnetic fields provide closely analogous equations for the divergence of stress, electric displacement and magnetic induction: 


$$
\begin{gathered}
\sigma_{i j, j}=-b_{i} \\
D_{i, i}=q \\
B_{i, i}=0
\end{gathered}
$$

Here $b_{i}$ is the body force density including inertial forces, and $q$ is the free charge density. Indicial notation is used to indicate Cartesian tensors, with commas indicating spatial derivatives. In the absence of free charge and body forces Eqns. (1)-(3) become equivalent except for the second order tensorial nature of stress contrasting with electric displacement and magnetic induction, which are vectors. It should be noted that while ferroelectrics are always insulators, or at best weakly semi-conducting, ferromagnets and ferroelastic shape memory alloys are commonly conducting. Therefore care should be taken in the treatment of free charge in composites containing these materials. Theories of magneto-electro-elastic materials nevertheless often neglect free charge as this provides a convenient formulation.

Further relations allow the conjugate quantities $(\varepsilon, D, H)$ to be expressed as gradients, defining strain as the symmetrized gradient of displacement $u_{i}$,

$$
\varepsilon_{i j}=\left(u_{i, j}+u_{j, i}\right) / 2
$$

and noting that for quasi-static conditions both electric and magnetic fields are irrotational, in consequence of Maxell's equations:

$$
\begin{gathered}
E_{i}=-\phi_{, i} \\
H_{i}=-\varphi_{, i}
\end{gathered}
$$

Here $\phi$ is the electric potential and $\varphi$ the magnetic potential. So far, the mechanical, electrical and magnetic equations are closely analogous. We next turn to the definitions of the remanent quantities associated with ferroic order. These variables indicate the material state in the absence of external loads. Here it is desirable to decompose the strain into a reversible part $\varepsilon_{i j}^{\text {rev }}$ due to the application of loads (which may be mechanical, electrical or magnetic loads), and a remanent part due to ferroelastic ordering. Then

$$
\varepsilon_{i j}=\varepsilon_{i j}^{\mathrm{rev}}+\varepsilon_{i j}^{\mathrm{R}}
$$


The analogous gradient variable in ferroelectricity is the electric field as defined in Eqn. (5). However, ferroic ordering does not provide a permanent electric field, but instead a polarization that is the moment of charge resulting from structural displacement of ions and polarization of individual ions. The polarization contributes to the total electric displacement. Hence, naming the reversible part of electric displacement $D_{i}^{\text {rev }}$ we could write:

$$
D_{i}=D_{i}^{\mathrm{rev}}+P_{i}^{\mathrm{R}}
$$

However, the conventional concept of material polarization includes all of the electric displacement that is due to the presence of the material, excepting only that part which would be present in vacuum with the same electric field, thus

$$
D_{i}=\varepsilon_{0} E_{i}+P_{i}
$$

where $\varepsilon_{0}$ is the permittivity of free space. The polarization so defined includes both the remanent part of polarization, $P_{i}^{\mathrm{R}}$, and that part of $D_{i}^{\mathrm{rev}}$ due to the presence of the material itself - that is all except the part due to the permittivity of free space.

For magnetic materials, it is conventional to make a similar division with magnetic field and magnetization, in the form:

$$
B_{i}=\mu_{0}\left(H_{i}+M_{i}\right)
$$

where $\mu_{0}$ is the permeability of free space. However, there is no analogous expression for stress and strain because they are unrelated (or undefined) in free space.

Constitutive relations for the materials may be developed by considering energy. An increment of internal energy $U$ is produced when external loads do work on the material:

$$
d U=\sigma_{i j} d \varepsilon_{i j}+E_{i} d D_{i}+H_{i} d B_{i}
$$

Notice that positive work increments are driven by stress (which is divergence free) changing the strain (which is a gradient variable) but also by electric field and magnetic field (both are gradient variables) changing the electric displacement and magnetic induction (divergence free). This positive work description thus produces a mixture of gradient terms and divergence free 
terms as the independent variables which is inconvenient for solving boundary value problems. A commonly used alternative (Alshits et al., 1992) is achieved through the Legendre transform

$$
\Psi=U-E_{i} D_{i}-H_{i} B_{i}
$$

such that

$$
d \Psi=\sigma_{i j} d \varepsilon_{i j}-D_{i} d E_{i}-B_{i} d H_{i}
$$

Taking $\Psi$, known as the electromagnetic enthalpy (Soh and Liu, 2005), to be a function of $\varepsilon_{i j}$, $E_{i}$ and $H_{i}$, the stress, electric displacement and magnetic induction may be derived as

$$
\sigma_{i j}=\frac{\partial \Psi}{\partial \varepsilon_{i j}} ; \quad D_{i}=-\frac{\partial \Psi}{\partial E_{i}} ; \quad B_{i}=-\frac{\partial \Psi}{\partial H_{i}}
$$

Expanding $\Psi$ as a power series and showing second order terms gives

$$
\Psi=\frac{1}{2} C_{i j k l} \varepsilon_{i j} \varepsilon_{k l}+\frac{1}{2} \kappa_{i j} E_{i} E_{j}+\frac{1}{2} \mu_{i j} H_{i} H_{j}-e_{i k l} E_{i} \varepsilon_{k l}-q_{i k l} H_{i} \varepsilon_{k l}-\lambda_{i k} E_{i} H_{k}+\cdots
$$

The linear material coefficients are $C_{i j k l}$, the elastic modulus, $\kappa_{i j}$, the dielectric permittivity tensor, $\mu_{i j}$, the magnetic permeability tensor, $e_{i k l}$, the piezoelectric tensor, $q_{i k l}$, the piezomagnetic tensor, and $\lambda_{i k}$ the magnetoelectric tensor.

From Eqn. (15) can be derived, for small changes $\varepsilon_{i j}, E_{i}$ and $H_{i}$ from a reference state, the linear constitutive relations

$$
\begin{gathered}
\sigma_{i j}=C_{i j k l} \varepsilon_{k l}-e_{k i j} E_{k}-q_{k i j} H_{k} \\
D_{i}=e_{i k l} \varepsilon_{k l}+\kappa_{i k} E_{k}+\lambda_{i k} H_{k} \\
B_{i}=q_{i k l} \varepsilon_{k l}+\lambda_{i k} E_{k}+\mu_{i k} H_{k}
\end{gathered}
$$

The appearance of minus signs in Eqn. (15) is somewhat arbitrary but the nonsymmetric form of (16)-(18) is not. However, expressing the electric and magnetic fields as gradients produces a symmetrized form that can be represented by the compact notation

$$
\Sigma_{i J}=L_{i J K l} Z_{K l} \quad(i, l=1 \ldots 3 ; J, K=1 \ldots .5)
$$


where $\Sigma_{i J}$ contains the elements of $\sigma_{i j}$ for $J=1,2,3, D_{i}$ for $J=4$ and $B_{i}$ when $J=5$. Similarly $Z_{K l}$ contains the elements of $\varepsilon_{k l}$ for $K=1,2,3, \phi_{, l}$ for $K=4$ and $\varphi_{, l}$ when $K=5$. Summation over the indices is conducted for $i, l=1 \ldots 3$ and $J, K=1 \ldots 5$. Then $L_{i J K l}$ is symmetric and contains the various material coefficients.

\section{Eshelby tensor and estimates of composite properties}

The Eshelby tensor (Eshelby, 1957) relates the transformation strain of a transforming ellipsoidal inclusion to the strain experienced by the inclusion when constrained by surrounding matrix material. Thus for an elastic, transforming medium undergoing a stress-free transformation strain (or eigenstrain) $\varepsilon_{i j}^{T}$ the total strain experienced by a transforming ellipsoidal inclusion embedded in a surrounding matrix of the same material is

$$
\varepsilon_{i j}=S_{i j k l} \varepsilon_{k l}^{\mathrm{T}}
$$

Eshelby showed that the strain is uniform within the inclusion. The coupled problem including electrical terms (Wang, 1992; Dunn and Taya, 1993) and magnetic terms (Li and Dunn, 1998; Li, 2000) is analogous, allowing a fully coupled Eshelby tensor to be defined in the form:

$$
Z_{K l}=S_{K l M n}^{\mathrm{MEE}} Z_{M n}^{\mathrm{T}}
$$

where the superscript MEE identifies the Eshelby tensor for Magneto-electro-elastic materials. Methods for computing the components of $S_{K I M n}^{\mathrm{MEE}}$ and applications have given by several authors, following procedures analogous to those of Eshelby (Huang and Kuo, 1997; Huang et al. 1998).

The significance of the Eshelby tensor for estimating the properties of composites arises because by varying the material properties and proportions of the ellipsoid, the interaction of diverse forms of composite phases with the surrounding medium can be estimated. Cylindrical fibres, spherical inclusions and laminar platelets are all special cases of ellipsoidal inclusions. Hence the average properties of composites can be estimated. 
A simple but powerful means of estimating composite properties is provided by the selfconsistent estimate (Hill, 1965). In the case of composites of ferroic or multiferroic phases, the self-consistent estimate arises by treating each phase of the composite as an inclusion embedded in a matrix that has the average properties of the overall composite. Each individual phase (or inclusion) has its own moduli, so that locally:

$$
\Sigma_{i J}=L_{i J K l} Z_{K l}
$$

while, denoting volume averages over the whole composite with an overbar, and the overall properties of the composite by $L_{i J K l}^{\circ}$,

$$
\bar{\Sigma}_{i J}=L_{i J K l}^{\mathrm{o}} \bar{Z}_{K l}
$$

Any deviation $\Sigma_{i J}-\bar{\Sigma}_{i J}$ in the local fields is related to the corresponding deviation $Z_{K l}-\bar{Z}_{K l}$ using the Eshelby inclusion model. This prescribes that for ellipsoidal inclusions in a matrix with overall properties $L_{i J K l}^{\mathrm{o}}$

$$
\Sigma_{i J}-\bar{\Sigma}_{i J}=-L_{i J K l}^{\mathrm{o}}\left(I_{i J K l}-\left(S_{K I M n}^{\mathrm{o}}\right)^{-1}\right)\left(Z_{K l}-\bar{Z}_{K l}\right)=-L_{i J K l}^{*}\left(Z_{K l}-\bar{Z}_{K l}\right)
$$

Where $I_{i J K l}$ is a collection of identity tensors defined such that $\mathbf{L I}=\mathbf{I} \mathbf{L}=\mathbf{L}$ and the inverse $\left(S_{K I M n}^{o}\right)^{-1}$ is chosen such that $\mathbf{S}^{-1} \mathbf{S}=\mathbf{S S}^{-1}=\mathbf{I}$. Eqn. (24) also defines the constraint tensor $L_{i J K l}^{*}$. Combining Eqns. (22), (23) and (24) provides the relationship

$$
\left(\mathbf{L}+\mathbf{L}^{*}\right) \mathbf{Z}=\left(\mathbf{L}^{\mathrm{o}}+\mathbf{L}^{*}\right) \overline{\mathbf{Z}}
$$

where indices have been omitted for brevity. Hence

$$
\boldsymbol{\Sigma}=\mathbf{L}\left(\mathbf{L}+\mathbf{L}^{*}\right)^{-1}\left(\mathbf{L}^{\mathrm{o}}+\mathbf{L}^{*}\right) \overline{\mathbf{Z}}
$$

Averaging over all inclusions and hence over the entire volume of the composite gives

$$
\overline{\mathbf{\Sigma}}=\left(\overline{\mathbf{L}\left(\mathbf{L}+\mathbf{L}^{*}\right)^{-1}\left(\mathbf{L}^{\mathrm{o}}+\mathbf{L}^{*}\right)}\right) \overline{\mathbf{Z}}
$$

Thus the overall properties $\mathbf{L}^{\mathrm{o}}$ of the composite could be estimated by the average

$$
\mathbf{L}^{\mathrm{o}}=\overline{\mathbf{L}\left(\mathbf{L}+\mathbf{L}^{*}\right)^{-1}\left(\mathbf{L}^{\mathrm{o}}+\mathbf{L}^{*}\right)}
$$


but for the inconvenient fact that both $\mathbf{L}^{0}$ itself and $\mathbf{L}^{*}$, which depends on $\mathbf{L}^{0}$ through the Eshelby tensor, appear on the right-hand side. All is not lost as $\mathbf{L}^{0}$ can still be computed by using a simple first guess such as $\overline{\mathbf{L}}$ and iteration of Eqn. (28) until a consistent set of moduli for the composite is found. This self-consistent estimate works well under a wide range of conditions.

An alternative approach, also based on the mean stress and strain fields in the composite, and valued for its close relationship with bounds derived from variational approaches, is the mean field method of Mori and Tanaka (Mori and Tanaka, 1973; Weng, 1990). The application to ferroic materials with magneto-electro-elastic coupling has been explored by several authors (Huang and Kuo, 1997; Lee et al., 2005). The Mori-Tanaka method is generally developed for composites with an identifiable matrix within which there is a volume fraction $f$ of inhomogeneities that modify the mean-fields. Consideration of the disturbance to the mean field caused by inhomogeneities leads to the estimate

$$
\mathbf{L}^{\mathrm{o}}=\mathbf{L}^{\mathrm{m}}\left(\mathbf{I}+f \mathbf{V}^{-1}\left(\mathbf{L}-\mathbf{L}^{\mathrm{m}}\right)\right)
$$

where $\mathbf{L}^{\mathrm{o}}$ is the overall tensor of moduli of the composite, $\mathbf{L}^{\mathrm{m}}$ and $\mathbf{L}$ are the corresponding moduli in the matrix and inhomogeneities respectively, and $\mathbf{V}$ is given by (Huang and Kuo, 1997):

$$
\mathbf{V}=(1-f)\left(\mathbf{L}-\mathbf{L}^{\mathrm{m}}\right) \mathbf{S}+\mathbf{L}^{\mathrm{m}}
$$

The Mori-Tanaka method has the advantage of providing explicit expressions for the moduli of the composite, and has been shown to agree well with generalized self-consistent methods and variational bounds for a range of material properties and inclusion geometries (Christensen, 1990).

The estimates of the linear properties of composites discussed so far are of use for a wide range of ferroic materials, and indicate, for example, that strong magnetoelectric coupling can be produced in certain composites, even when none of the individual phases has such coupling. However, these methods do not deal with the remanent states produced by the symmetry related ferroic crystal variants, which will be considered in the remaining sections. 


\section{Ferroic crystal variants and domains}

In a typical ferroic material, the transformation from a high symmetry state to a lower symmetry state with multiple, symmetry-related crystal variants produces the ferroic properties. For example, the ferroelectric barium titanate, $\mathrm{BaTiO}_{3}$, has cubic perovskite crystal structure above the Curie temperature, but has tetragonal structure immediately below the Curie temperature. The tetragonal state has a spontaneous polarization of each unit cell, along the crystallographic caxis, in the absence of externally applied electric fields. Similarly, the magnetic shape memory alloy $\mathrm{Ni}_{2} \mathrm{MnGa}$ transforms from cubic to tetragonal and develops spontaneous magnetization along the c-axis. While several shape memory alloys exhibit cubic-tetragonal transformations, a wide variety of other crystal systems are found (James and Hane, 2000). Among the most widely used shape memory alloys are the nickel-titanium alloys exhibiting a cubic-monoclinic transformation. Nevertheless, the tetragonal system is instructive and relative easy to study, and so will be used in examples here.

In most general terms, we can consider a ferroic material with magneto-electro-elastic linear properties, and capability for all three ferroic orders, resulting in spontaneous strain, polarization and magnetization. Pure single phase materials with all of these properties are rare, but by developing the theory most generally the relations between the different types of materials found in practice will become evident. Let the low symmetry phase have $N$ distinct variants, $I=1 \ldots N$, each with its own spontaneous strain $\boldsymbol{\varepsilon}^{I}$, spontaneous polarization $\mathbf{P}^{I}$ and magnetization $\mathbf{M}^{I}$. Then in a region of material comprising the $I$ th variant, the linear constitutive Eqns. (16)-(18) can be modified to read:

$$
\begin{gathered}
\sigma_{i j}=C_{i j k l}\left(\varepsilon_{k l}-\varepsilon_{k l}^{I}\right)-e_{k i j} E_{k}-q_{k i j} H_{k} \\
D_{i}-P_{i}^{I}=e_{i k l}\left(\varepsilon_{k l}-\varepsilon_{k l}^{I}\right)+\kappa_{i k} E_{k}+\lambda_{i k} H_{k} \\
B_{i}-\mu_{i k} M_{k}^{I}=q_{i k l}\left(\varepsilon_{k l}-\varepsilon_{k l}^{I}\right)+\lambda_{i k} E_{k}+\mu_{i k} H_{k}
\end{gathered}
$$

At first sight it may appear that the crystal variants could be randomly distributed over the volume of a ferroic crystal with free variation from unit cell to unit cell. Such a balanced distribution of spontaneous states could reduce energy by avoiding the generation of a macroscopic strain, polarization or magnetization. However, ferroic ordering arises when, as is 
commonly the case, there is also an energy penalty associated with adjacent cells having different values of the spontaneous state. This energy penalty is due to elastic energy in the ferroelastic case, dipole-dipole interaction energy for ferroelectrics, and exchange energy in magnetic materials. There is then competition between a macroscopic driving force towards a balanced average and a microscopic driving force towards uniformity. The result is the formation of domains that are regions of uniform spontaneous strain, polarization or magnetization (Tagantsev et al. 2010). The size and form of these domains is determined by energetic considerations and varies greatly, but the appearance of domain patterns is so widespread as to be considered a defining feature of ferroics (Van Aken et al., 2007). Since the domains can comprise distinct crystal variants, a ferroic material can be thought of as a composite of domains.

It is instructive to consider the conditions that pertain on the interfaces between adjacent domains. These interfaces are known as domain walls when they separate domains within a single phase. Fig. 2 shows an interface, which may generally be curved, with local normal direction $\mathbf{n}$, separating domains numbered 1 and 2. Then, in the absence of body forces or charges, the governing Eqns. (1)-(3) imply that the local stress, electric displacement and magnetic induction must have no jump in normal component:

$$
\left(\Sigma_{i J}^{1}-\Sigma_{i J}^{2}\right) n_{i}=0
$$

Similarly, the irrotational nature of the gradient terms requires that

$$
\left(Z_{K l}^{1}-\Sigma_{K l}^{2}\right) t_{l}=0
$$

Where $t_{l}$ is any vector tangent to the interface, such that $n_{l} t_{l}=0$. Eqns. (34) and (35) can be used to specify compatibility conditions for the interface in terms of the spontaneous state of the crystal variants $\left(\boldsymbol{\varepsilon}^{I}, \mathbf{P}^{I}, \mathbf{M}^{I}\right)$ for minimum energy. The pair of crystal variants meets with a minimum energy arrangement if the elastic stress, electric field and magnetic field are zero in both variants. Using this condition and Eqns. (31)-(33) results in

$$
\begin{gathered}
\boldsymbol{\varepsilon}=\boldsymbol{\varepsilon}^{I} \\
\mathbf{D}=\mathbf{P}^{I}
\end{gathered}
$$




$$
\mathbf{B}=\boldsymbol{\mu} \mathbf{M}^{I}
$$

meaning that each variant adopts one of the $N$ undisturbed spontaneous states.

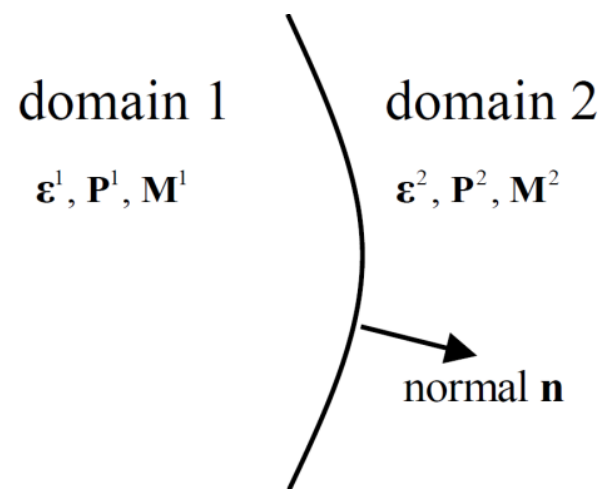

Fig. 2 The interface between a pair of ferroic domains.

Of course, the zero-field condition may not be possible in the presence of external loading, or there may be no spatial arrangement of the crystal variants that can achieve this condition. However, in the absence of applied loading, and given that there are many possible arrangements the material might adopt, it is reasonable to suppose that Eqns. (36)-(38) may hold pointwise if there is an arrangement which allows it. This is to treat the mixture of crystal variants as a minimizer of energy. Using Eqns. (34) and (35), the consequences of (36)-(38) may be written as:

$$
\begin{gathered}
\left(\boldsymbol{\varepsilon}^{1}-\boldsymbol{\varepsilon}^{2}\right) \cdot \mathbf{t}=0 \\
\left(\mathbf{P}^{1}-\mathbf{P}^{2}\right) \cdot \mathbf{n}=0 \\
\left(\mathbf{M}^{1}-\mathbf{M}^{2}\right) \cdot \mathbf{n}=0
\end{gathered}
$$

In Eqn. (41) it has been assumed that the magnetic permeability tensor $\boldsymbol{\mu}$ is the same in both domains; more generally it is the product $\boldsymbol{\mu} \mathbf{M}$ whose normal component is continuous. Eqn. (39) can conveniently be rewritten in terms of the interface normal $\mathbf{n}$ as

$$
\boldsymbol{\varepsilon}^{1}-\boldsymbol{\varepsilon}^{2}=\mathbf{n} \otimes \mathbf{a}+\mathbf{a} \otimes \mathbf{n}
$$

where $\mathbf{a}$ can be chosen as any vector to satisfy the equation and $\mathbf{n} \otimes \mathbf{a}$ indicates the outer product $n_{i} a_{j}$. Eqn. (42) is the well-known Hadamard condition for continuity of displacement at an 
interface, and (40)-(42) collectively provide compatibility conditions for an interface between low energy domains in ferroic materials. The consequences of these conditions have been widely explored in the various types of ferroic material (Ball and James, 1987; Bhattacharya, 2003; Shu and Bhattacharya, 2001, Tsou and Huber, 2010; De Simone and James, 2002).

It is worth noting that, in the presence of stress, electric field and magnetic field, the invariants and principal directions of both the strain and polarization can be modified. This arises because both quantities are structural in nature, being affected by the location and arrangement of atoms or ions. Magnetization, however, typically has a fixed or very nearly fixed magnitude, determined by spin states. Then only the direction of magnetization can be varied away from one of the spontaneous states at an energy cost known as the magnetocrystalline anisotropy energy.

Suppose that the spontaneous states of the crystal variants are known. Then, for a particular pair of crystal variants meeting at an interface, Eqns. (40)-(42) specify conditions for an interface orientation that can give minimum energy of the domains. Taking account of the symmetry of the strain tensor, (40)-(42) provide 12 equations for the 3 components of $\mathbf{n}$, and so the problem of finding a low energy interface is strongly overdetermined: at first sight it may seem unlikely that any such interface can form. However, in most ferroic phases, the symmetry related crystal variants do admit low energy domain arrangements. It has been shown (Ball and James, 1987) that Eqn. (42) may have no solutions, or at most two distinct solutions for $\mathbf{n}$. It very often arises that one of these solutions will satisfy Eqn. (40) or (41). Thus it is often the case that ferroic domains in materials with ferroelastic ordering are separated by planar domain walls with a welldefined orientation. The case where $\boldsymbol{\varepsilon}^{1}=\boldsymbol{\varepsilon}^{2}$, such that Eqn. (42) is trivially satisfied for any $\mathbf{n}$, is also of importance in ferromagnetic and ferroelectric materials. Then the domain wall orientation is constrained only by Eqn. (40) or (41) which are easily satisfied, giving rise to a continuous set of solutions for $\mathbf{n}$ in the plane normal to $\mathbf{P}^{1}-\mathbf{P}^{2}$ or $\mathbf{M}^{1}-\mathbf{M}^{2}$. The resulting domain walls are wavy, developable surfaces commonly found in both ferroelectrics and ferromagnetic materials.

The domain walls themselves comprise narrow regions of material with steep gradients in some combination of strain, electrical polarization and magnetization. They have finite width, again determined by energetic considerations. In ferroelastic materials and ferroelectrics, domain walls can be almost atomically sharp and widths less than 10nm are common. Ferromagnetic domain 
walls are usually thicker, 10-100nm being typical. Provided that the domain size is much greater than this, it is common to neglect the domain wall energy, so that Eqns. (40)-(42) can be treated as the main constraint on the formation of domain patterns.

The presence of domain pairs whose domain walls have unique orientation in ferroics has far reaching consequences. A mixture of such domains can then take on only one form, namely alternating layers of the two crystal variants in the form of a laminate. Closer consideration of such laminates is given in sections 4 and 5 .

For the moment, let us explore further the consequences of the kind of field-free minimum energy patterns of domains for which Eqns. (36)-(38) hold. Consider now deforming the outer surface of a body of ferroic material occupying a volume $V$ into a shape consistent with uniform straining. That is, for each point $\mathbf{x}$ on the surface, the displacement $\mathbf{u}(\mathbf{x})$ should be

$$
u_{i}(\mathbf{x})=\varepsilon_{i j}^{\mathrm{o}} x_{j}
$$

for some overall imposed strain $\varepsilon_{i j}^{\mathrm{o}}$. It is easy to show that the pointwise strain then averages to $\varepsilon_{i j}^{\mathrm{o}}$, that is

$$
\bar{\varepsilon}_{i j}=\frac{1}{V} \int_{V} \varepsilon_{i j}(\mathbf{x}) \mathrm{d} V=\varepsilon_{i j}^{\mathrm{o}}
$$

Thus an average strain can be imposed by controlling surface displacements. Similar arguments apply to electric field, which can be controlled using surface voltages. However it is not straightforward to impose an average electric displacement or magnetic induction; care must be then be taken to account for the space outside $V$. In the case of electric displacement, a uniform $D_{i}^{\mathrm{o}}$ can be compensated at the boundary of $V$ by placing free charge on the boundary with density $D_{i}^{\mathrm{o}} n_{i}^{\mathrm{V}}$ where $n_{i}^{\mathrm{V}}$ is the outward surface normal of $V$. The external electric displacement is then zero. However, imposing this charge density on the surface of $V$ does not guarantee $\bar{D}_{i}=D_{i}^{\mathrm{o}}$ because there could be electric fields both internal and external to $V$. This contrasts with the mechanical case, where external stresses can often be assumed to be zero. If the material can adopt a polarization state that matches $D_{i}^{\circ}$ at no cost in energy, then this state minimizes energy overall by eliminating electric field. The magnetic case is less straightforward as there is 
no magnetic analogue for charge. An external magnetic field $H_{i}^{\mathrm{o}}$ can be imposed outside $V$. Once again, if the material can adopt a magnetization state aligned with $H_{i}^{\mathrm{o}}$ this can minimize energy. There thus arises the question of what states of average strain, polarization and magnetization can be achieved by mixtures of the crystal variants within $V$. If Eqns. (36)-(38) hold, and neglecting inhomogeneity in $\boldsymbol{\mu}$, the macroscopic strain, polarization and magnetization are given by simple volume averages. Then, regardless of the detailed arrangement of the crystal variants, it is only their overall volume fractions $f^{I}$ that affect the average, giving

$$
\begin{aligned}
\boldsymbol{\varepsilon}^{\mathrm{o}} & =\sum_{I=1}^{N} f^{I} \boldsymbol{\varepsilon}^{I} \\
\mathbf{P}^{\mathrm{o}} & =\sum_{I=1}^{N} f^{I} \mathbf{P}^{I} \\
\mathbf{M}^{\mathrm{o}} & =\sum_{I=1}^{N} f^{I} \mathbf{M}^{I}
\end{aligned}
$$

To make the discussion more concrete, take the example of the magnetic shape memory alloy $\mathrm{Ni}_{2} \mathrm{MnGa}$. The martensitic phase has tetragonal structure with $c<a$ and magnetization aligned with the $\pm c$ axis (Webster et al., 1984; Pons et al., 2000; Kiefer and Lagoudas, 2005). The variants are thus 6 in number with the spontaneous magnetization states

$$
\mathbf{M}^{1}=m_{0} \mathbf{e}_{1} ; \quad \mathbf{M}^{2}=-m_{0} \mathbf{e}_{1} ; \quad \mathbf{M}^{3}=m_{0} \mathbf{e}_{2} ; \quad \mathbf{M}^{4}=-m_{0} \mathbf{e}_{2} ; \quad \mathbf{M}^{5}=m_{0} \mathbf{e}_{3} ; \quad \mathbf{M}^{6}=m_{0} \mathbf{e}_{3}
$$

where $m_{0}$ is the magnitude of spontaneous magnetization and $\mathbf{e}_{1}, \mathbf{e}_{2}, \mathbf{e}_{3}$ are unit vectors along the crystallographic axes. The corresponding spontaneous strain states are

$$
\begin{aligned}
& \boldsymbol{\varepsilon}^{1}=\boldsymbol{\varepsilon}^{2}=\mathbf{I} \varepsilon_{a}+\left(\varepsilon_{c}-\varepsilon_{a}\right)\left(\mathbf{e}_{1} \otimes \mathbf{e}_{1}\right) \\
& \boldsymbol{\varepsilon}^{3}=\boldsymbol{\varepsilon}^{4}=\mathbf{I} \varepsilon_{a}+\left(\varepsilon_{c}-\varepsilon_{a}\right)\left(\mathbf{e}_{2} \otimes \mathbf{e}_{2}\right) \\
& \boldsymbol{\varepsilon}^{5}=\boldsymbol{\varepsilon}^{6}=\mathbf{I} \varepsilon_{a}+\left(\varepsilon_{c}-\varepsilon_{a}\right)\left(\mathbf{e}_{3} \otimes \mathbf{e}_{3}\right)
\end{aligned}
$$

where $\mathbf{I}$ is the $3 \times 3$ identity matrix, $\varepsilon_{a}$ is the (positive) $a$-axis strain, and $\varepsilon_{c}$ is the (negative) $c$ axis strain. The alloy is not ferroelectric. The six variants are illustrated schematically in Fig. 3. 


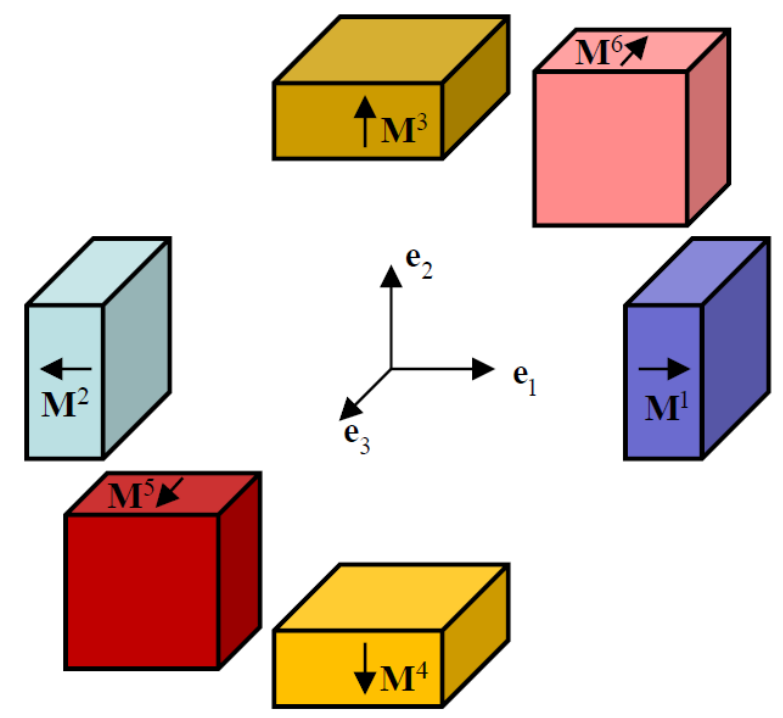

Fig. 3. Tetragonal martensite variants with magnetization aligned to the $c$-axis.

Given a macroscopic magnetization $\mathbf{M}^{\mathrm{o}}$, and strain $\boldsymbol{\varepsilon}^{\circ}$, is there a set of volume fractions $f^{I}$ of the 6 variants that will achieve this average by a composite of domains? This is a constrained linear algebra problem. Due to the symmetry of strain, Eqn. (45) provides 6 linear equations and Eqn. (47) provides a further 3. Additionally,

$$
\sum f^{I}=1
$$

as the volume fractions must sum to unity, providing one further equation. Since, in the tetragonal case, the strain matrices are all diagonal in form, any off-axis terms in $\boldsymbol{\varepsilon}^{\mathrm{o}}$ must be zero. Furthermore, the variants each have the same volume of unit cell, so that $\varepsilon_{11}^{\mathrm{o}}+\varepsilon_{22}^{\mathrm{o}}+\varepsilon_{33}^{\mathrm{o}}=\varepsilon_{c}+2 \varepsilon_{a}$ is required. Assuming that these conditions on $\boldsymbol{\varepsilon}^{\mathrm{o}}$ are satisfied, there remain only two independent equations derived from Eqn. (45), so that there are in total 6 equations for the 6 unknown $f^{I}$ : 


$$
\left[\begin{array}{cccccc}
\varepsilon_{c} & \varepsilon_{c} & \varepsilon_{a} & \varepsilon_{a} & \varepsilon_{a} & \varepsilon_{a} \\
\varepsilon_{a} & \varepsilon_{a} & \varepsilon_{c} & \varepsilon_{c} & \varepsilon_{a} & \varepsilon_{a} \\
m_{0} & -m_{0} & 0 & 0 & 0 & 0 \\
0 & 0 & m_{0} & -m_{0} & 0 & 0 \\
0 & 0 & 0 & 0 & m_{0} & -m_{0} \\
1 & 1 & 1 & 1 & 1 & 1
\end{array}\right]\left[\begin{array}{c}
f^{1} \\
f^{2} \\
f^{3} \\
f^{4} \\
f^{5} \\
f^{6}
\end{array}\right]=\left[\begin{array}{c}
\varepsilon_{11}^{\mathrm{o}} \\
\varepsilon_{22}^{\mathrm{o}} \\
M_{1}^{\mathrm{o}} \\
M_{2}^{\mathrm{o}} \\
M_{3}^{\mathrm{o}} \\
1
\end{array}\right]
$$

The constraint $f^{I} \geq 0$ must be added. The resulting system of equations has solutions provided that the prescribed macroscopic state $\left(\mathbf{M}^{\mathrm{o}}, \boldsymbol{\varepsilon}^{\mathrm{o}}\right)$ lies within the convex hull determined by the spontaneous states of the set of variants. In the case illustrated by Eqn. (53) a unique set of $f^{I}$ can be found for a given $\left(\mathbf{M}^{\circ}, \boldsymbol{\varepsilon}^{\mathrm{o}}\right)$, but more generally the $f^{I}$ may not be unique, so that a prescribed macroscopic state could be achieved with different volume fractions, see Tsou and Huber (2010) for further discussion. Of course, if $\left(\mathbf{M}^{\mathrm{o}}, \boldsymbol{\varepsilon}^{\mathrm{o}}\right)$ is outside the convex hull there is no solution.

What can be said about the linear elastic, electrical and magnetic properties of the composite of domains? Knowledge of the volume fractions will provide only bounds on the various moduli, and these may be far apart; estimates could also be made using the self-consistent or MoriTanaka schemes. However, a specific evaluation of the moduli would require knowledge of how the domains were arranged. So we may ask: once the volume fractions are known, is there a specific arrangement of domains that satisfies the compatibility conditions? This question is discussed further in section 5.

\section{Laminates of ferroic crystal variants}

A mixture of two crystal variants with a unique orientation of interface normal, as shown in Fig. 4, is a laminate. This pattern of microstructure is widespread in ferroics, and there have been many studies of the properties of ferroic laminates (Kessler and Balke, 2001, Weng and Wong, 2009; Li and Liu, 2004, Li and Ma, 2008, De Simone and James, 2002; Tsou et al., 2013) including estimates of the elastic, electrical and magnetic moduli. 


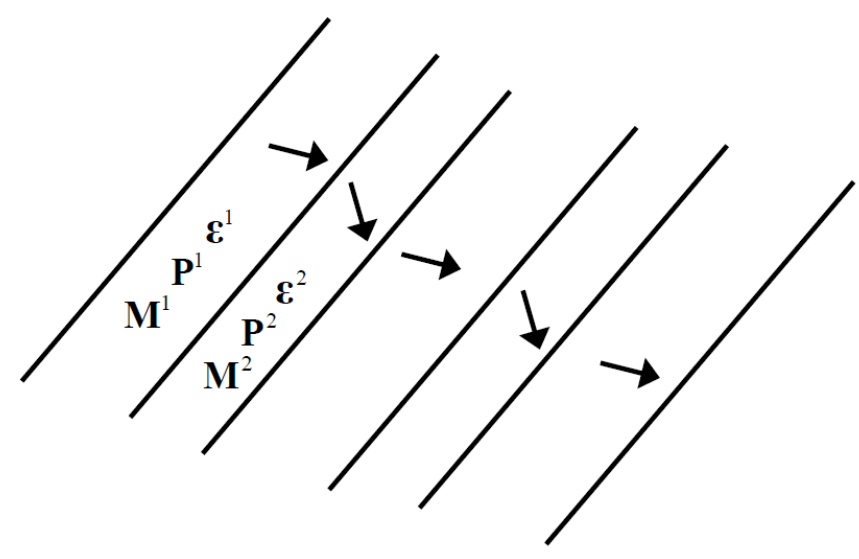

Fig 4. A laminate comprising alternating layers of two crystal variants.

Next, following a procedure similar to that of Li and Liu (2004), consider the magneto-electroelastic moduli of such a composite of domains. The laminate structure enforces continuity of stress, electric displacement and magnetic induction components normal to the interfaces, as well as strain, electric field and magnetic field components tangential to the interfaces. This allows a straightforward and exact evaluation of the moduli of the composite. First, rotate coordinates into a system where the $x_{3}$ coordinate axis is normal to the interface. In this coordinate system, the linear constitutive equation, Eqn. (19), for each crystal variant, and for the composite of layers, can be rewritten in the form

$$
\left[\begin{array}{c}
\boldsymbol{\Sigma}^{\perp} \\
\boldsymbol{\Sigma}^{\|}
\end{array}\right]=\left[\begin{array}{cc}
\mathbf{P} & \mathbf{Q} \\
\mathbf{Q}^{\mathrm{T}} & \mathbf{R}
\end{array}\right]\left[\begin{array}{c}
\mathbf{Z}^{\perp} \\
\mathbf{Z}^{\|}
\end{array}\right]
$$

where $\boldsymbol{\Sigma}^{\perp}=\left[\sigma_{31}, \sigma_{32}, \sigma_{33}, D_{3}, B_{3}\right]^{\mathrm{T}}$ contains the components of $\Sigma_{i J}$ which are continuous across the interface, and thus uniform in the laminate. Meanwhile $\boldsymbol{\Sigma}^{\|}$contains the remaining components of $\Sigma_{i J}$, which may be discontinuous at the interface. Similarly, $\mathbf{Z}^{\|}=\left[\varepsilon_{11}, \varepsilon_{22}, \varepsilon_{12},-E_{1},-E_{2},-H_{1},-H_{2}\right]^{\mathrm{T}}$ contains those components of $Z_{K l}$ which are continuous across the interface, and thus uniform in the laminate. The remaining components of $Z_{K l}$ are contained in $\mathbf{Z}^{\perp}$. The various components of $L_{i J K l}$ are arranged into the sub-matrices $\mathbf{P}, \mathbf{Q}$ and $\mathbf{R}$, which thus contain the material moduli for a crystal variant in the rotated coordinate system. 
We seek corresponding moduli $\mathbf{P}^{\mathrm{o}}, \mathbf{Q}^{\circ}$ and $\mathbf{R}^{\mathrm{o}}$ for the laminate. Let superscript 1 or 2 indicate the field variables in the first or second layer, respectively. Now, since $\boldsymbol{\Sigma}^{\perp 1}=\boldsymbol{\Sigma}^{\perp 2}=\boldsymbol{\Sigma}^{\perp}$ and $\mathbf{Z}^{\| 1}=\mathbf{Z}^{\| 2}=\mathbf{Z}^{\|}$, it can easily be shown that

$$
\mathbf{Z}^{\perp}=\overline{\mathbf{P}^{-1}} \boldsymbol{\Sigma}^{\perp}-\overline{\mathbf{P}^{-1} \mathbf{Q}} \mathbf{Z}^{\|}
$$

and

$$
\boldsymbol{\Sigma}^{\|}=\overline{\mathbf{Q}^{\mathrm{T}} \mathbf{P}^{-1}} \boldsymbol{\Sigma}^{\perp}+\overline{\mathbf{R}-\mathbf{Q}^{\mathrm{T}} \mathbf{P}^{-1} \mathbf{Q}} \mathbf{Z}^{\|}
$$

Overbars indicate volume averaging over the two variants in the laminate. Inverting Eqn. (55) gives

$$
\boldsymbol{\Sigma}^{\perp}=\left(\overline{\mathbf{P}^{-1}}\right)^{-1} \mathbf{Z}^{\perp}+\left(\overline{\mathbf{P}^{-1}}\right)^{-1} \overline{\mathbf{P}^{-1} \mathbf{Q}} \mathbf{Z}^{\|}
$$

And substituting into Eqn. (56) gives

$$
\boldsymbol{\Sigma}^{\|}=\overline{\mathbf{Q}^{\mathrm{T}} \mathbf{P}^{-1}}\left(\overline{\mathbf{P}^{-1}}\right)^{-1} \mathbf{Z}^{\perp}+\left(\overline{\mathbf{Q}^{\mathrm{T}} \mathbf{P}^{-1}}\left(\overline{\mathbf{P}^{-1}}\right)^{-1} \overline{\mathbf{P}^{-1} \mathbf{Q}}+\overline{\mathbf{R}-\mathbf{Q}^{\mathrm{T}} \mathbf{P}^{-1} \mathbf{Q}}\right) \mathbf{Z}^{\|}
$$

From Eqns. (57) and (58) the overall properties of the laminate can be identified as

$$
\begin{gathered}
\mathbf{P}^{\mathrm{o}}=\left(\overline{\mathbf{P}^{-1}}\right)^{-1} \\
\mathbf{Q}^{\mathrm{o}}=\left(\overline{\mathbf{P}^{-1}}\right)^{-1} \overline{\mathbf{P}^{-1} \mathbf{Q}} \\
\mathbf{R}^{\mathrm{o}}=\overline{\mathbf{Q}^{\mathrm{T}} \mathbf{P}^{-1}}\left(\overline{\mathbf{P}^{-1}}\right)^{-1} \overline{\mathbf{P}^{-1} \mathbf{Q}}+\overline{\mathbf{R}-\mathbf{Q}^{\mathrm{T}} \mathbf{P}^{-1} \mathbf{Q}}
\end{gathered}
$$

Once $\mathbf{P}^{\circ}, \mathbf{Q}^{\circ}$ and $\mathbf{R}^{\circ}$ are found, it is a straightforward matter to reassemble $L_{i J K l}^{\circ}$ in the rotated coordinate system and then rotate back into global coordinates.

A remarkable feature of many ferroic crystals is that the material can form higher rank laminates by layering together regions of simple laminate. Examples of such higher rank laminates are illustrated in Fig. 5. In Fig. 5(a) the laminate is of rank-2 and is formed by layering together two simple laminates. The more complicated example in Fig. 5(b) is of rank-3, formed by layering together two distinct laminates, each of rank-2. A cube-shaped section of the rank-3 lamination is 
illustrated in perspective to show the complicated 3-dimensional arrangement of the layers. Using a hierarchical framework, arbitrarily complicated laminates can be analyzed mathematically. However, it is rare in practice to find laminations of rank greater than 3 in real materials.

The laminates in Fig. 5 show fairly coarse sub-layers within each layer for the purpose of illustration. However, the sub-layers can be much finer. If the sub-layers are many times finer than their resulting composite layers, each composite layer can be treated as a homogenized medium for the purposes of estimating overall properties. Eqns. (59)-(61) can then be used in a hierarchical procedure: First an estimate is made of the homogenized properties of a lamination made of individual crystal variants. Then, several such layers are each treated as the laminae building up a more complicated lamination. In this way, it is possible to estimate the moduli of an arbitrarily complex laminate. Note that the results are now only approximate: the sub-layers would need to be of vanishing thickness to make the estimate exact.

(a)

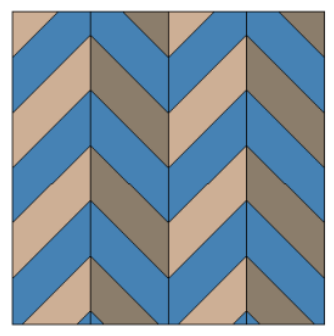

(b)

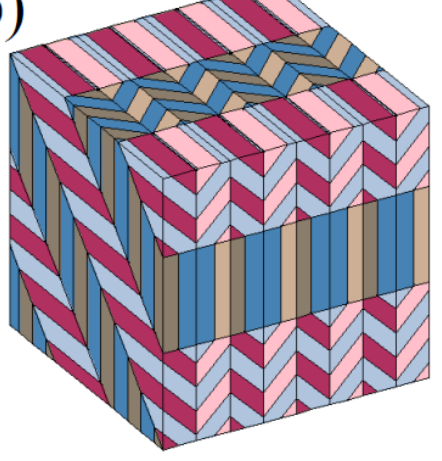

Fig. 5. Laminates of (a) rank-2 and (b) rank-3. Colours indicate distinct crystal variants.

In the context of the more complicated laminates of the kind shown in Fig. 5, it is of interest to explore whether the compatibility Eqns. (40) - (42) can be satisfied at the many interfaces that form. By construction, it can be shown that for every macroscopic state $\left(\boldsymbol{\varepsilon}^{\mathrm{o}}, \mathbf{P}^{\mathrm{o}}, \mathbf{M}^{\mathrm{o}}\right)$ that can be reached through Eqns. (45) - (47) with all volume fractions $f^{I}$ satisfying $\sum f^{I}=1$ and $f^{I} \geq 0$ (that is, for any macroscopic state in the convex hull of the spontaneous states of the variants) there is a pattern of lamination satisfying the compatibility conditions in at least an approximate, 
layer-wise manner. Specifically, the compatibility conditions will be satisfied for every interface in the finest laminations, and then layer-to-layer, treating each composite layer as a homogenized medium with an average state $(\overline{\boldsymbol{\varepsilon}}, \overline{\mathbf{P}}, \overline{\mathbf{M}})$. A construction that achieves compatibility in this way has been given for ferroelastic martensites by Bhattacharya (1993), and readily extends to ferroelectrics (Li and Liu, 2004) and ferromagnetics (De Simone and James, 2002). However, the problem of finding the set of macroscopic states that can be achieved while satisfying the compatibility conditions exactly at every interface remains unsolved.

\section{Applications in polycrystalline films}

In this section, a brief discussion is given of the application of the theory of compatibility to relaxed states in freestanding polycrystalline thin films of ferroic materials. Previous sections have dealt with composites of ferroic domains with a common crystallographic orientation. In a polycrystal, the grain-boundaries introduce interfaces that can have arbitrary orientation relative to the adjacent crystal lattices, and also allow arbitrary relative rotations of crystal axes between grains. Typically, the compatibility constraints on grains in random polycrystals are so severe that stress-free states are unlikely and complex energy-minimizing patterns form (Bhattacharya, 1993). However, in polycrystalline thin films and lamellae, the out-of-plane constraints can be relaxed, giving the material greater freedom to adopt a stress-free configuration (Shu, 2000). For simplicity, the example given here neglects constraint imposed by a substrate, consistent with the situation of a freestanding, unconstrained region of thin film. The methods are readily extended to other cases. Consider, then, the problem of finding a stress-free state in a ferroic lamella extracted as a section from a polycrystalline material. The theory will be developed here with reference to ferroelastic martensite.

First, examine a junction of grains in a ferroelastic lamina. A group of $N$ such grains, $i=1 \cdots N$, fit together around a junction in this thin lamella that is taken to lie in the $x-y$ plane. The grain boundary between pairs of grains $(i, j)$ has vector normal direction $\mathbf{n}_{i j}$ and tangential direction $\mathbf{t}_{i j}$ in the plane of the lamella. Here subscripts label only the grain numbers and are not tensorial indices - summation over repeated indices will not be implied unless stated explicitly. Each 
grain has its crystallographic axes rotated relative to reference $(x, y, z)$ coordinates by rotation matrix $\mathbf{R}_{i}$ and undergoes a transformation strain $\boldsymbol{\varepsilon}_{i}$ belonging to the set of stress-free spontaneous states, or mixtures of such martensitic variants. Fig. 6 illustrates such an arrangement of grains, with reference coordinates in grain number 1. Can the resulting group of grains fit together without stress? How will the individual grains organize their microstructure to minimize energy?

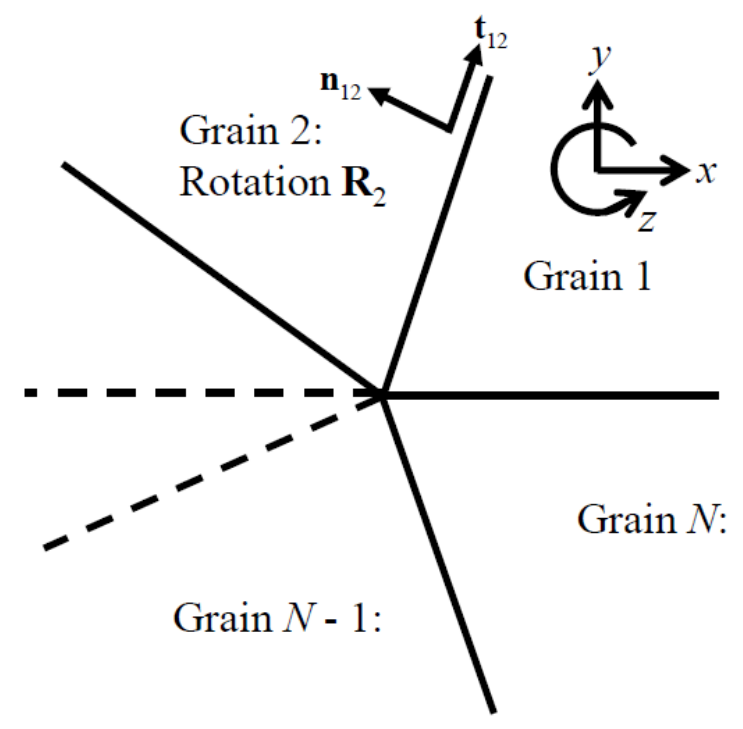

Fig. 6. Configuration of grains around a junction.

Suppose that the polycrystal is initially formed above the transformation temperature, but on cooling each grain undergoes a transformation strain that is uniform on the scale of the grain (though not on finer scales), with the grain comprising a mixture of the stress-free transformation strains that correspond to the spontaneous states of individual crystal variants. Then the transformation strain may be written, in the local coordinates of the grain, as

$$
\boldsymbol{\varepsilon}_{i}^{\prime}=\sum_{k} f_{i k} \boldsymbol{\varepsilon}_{k}^{0}
$$

where $\boldsymbol{\varepsilon}_{k}^{0}$ is the transformation strain of the $k$ th crystal variant and $f_{i k}$ is the volume fraction of that variant in the $i$ th grain. In reference coordinates, this becomes 


$$
\boldsymbol{\varepsilon}_{i}=\sum_{k} f_{i k} \mathbf{R}_{i} \boldsymbol{\varepsilon}_{k}^{0} \mathbf{R}_{i}^{\mathrm{T}}
$$

Where adjacent grains meet, the compatibility condition given by Eqn. (42),

$$
\boldsymbol{\varepsilon}_{i}-\boldsymbol{\varepsilon}_{j}=\mathbf{a}_{i j} \otimes \mathbf{n}_{i j}+\mathbf{n}_{i j} \otimes \mathbf{a}_{i j}
$$

can be used to ensure continuity of displacement. For materials that exhibit ferroelectricity or ferromagnetism, Eqns. (40) and (41) would also apply. Out-of-plane components of the electric displacement and magnetization may also be constrained depending on the surface conditions, or external conditions. If Eqn. (64) is satisfied for some choice of vector $\mathbf{a}_{i j}$, then the grain boundary is a compatible interface in the stress-free state. While Eqn. (64) is suitable for full three-dimensional constraint, the case of a thin lamella is less constrained provided that the grains are much larger than the lamellar thickness. This is because out-of-plane displacements are unconstrained. In fact, only a single displacement component is of relevance in the lamellar case: displacement in the plane of the lamella and in the direction along the grain boundary. Continuity requires no jump of displacement along the boundary as it is crossed. A condition for matching displacement components along the boundary is achieved by considering Eqn. (64) resolved in the $\mathbf{t}_{i j}$ direction:

$$
\mathbf{t}_{i j} \cdot\left(\boldsymbol{\varepsilon}_{i}-\boldsymbol{\varepsilon}_{j}\right) \cdot \mathbf{t}_{i j}=\mathbf{t}_{i j} \cdot\left(\mathbf{a}_{i j} \otimes \mathbf{n}_{i j}+\mathbf{n}_{i j} \otimes \mathbf{a}_{i j}\right) \cdot \mathbf{t}_{i j}=0
$$

The compatibility conditions for the grains of the lamella become a set of $N$ equations of the form

$$
\mathbf{t}_{12} \cdot \boldsymbol{\varepsilon}_{1} \cdot \mathbf{t}_{12}-\mathbf{t}_{12} \cdot \boldsymbol{\varepsilon}_{2} \cdot \mathbf{t}_{12}=0
$$

and so forth. Defining scalar $l_{i j k}$ as the linear strain in grain $i$ along the boundary with grain $j$ due to the $k$ th crystal variant,

$$
l_{i j k}=\mathbf{t}_{i j} \cdot\left(\mathbf{R}_{i} \boldsymbol{\varepsilon}_{k}^{0} \mathbf{R}_{i}^{\mathrm{T}}\right) \cdot \mathbf{t}_{i j}
$$

The $N$ compatibility conditions then take the form

$$
\sum_{k} f_{i k} l_{i j k}-f_{j k} l_{j i k}=0
$$


where only the $N$ pairings $(i, j)$ for which grain boundaries exist are included in the sum. Note that the formulation is readily extended to consider the more general problem of a group of grains in a polycrystalline lamella by allowing all $(i, j)$ pairings that represent grain boundaries present in the polycrystal. For the present problem, the equations entailed by (68) form a closed chain of connections between adjacent grains around a single junction, and ignore any constraint external to that group. Eqn. (68) expresses the compatibility conditions explicitly in terms of the volume fractions of the crystal variants, and the following additional constraints apply:

$$
\sum_{k} f_{i k}=1
$$

and

$$
f_{i k} \geq 0
$$

Eqn. (69) produces $N$ linear equations, and Eqn. (70) is a set of $m N$ inequalities in a system with $m$ crystal variants and $N$ grains. Eqns. (68) and (69) provide $2 N$ linear relations for the $m N$ volume fractions in the individual grains. Thus the system of linear equations is underdetermined except in the case $m=2$. For tetragonal martensite, $m=3$, and so there exists a space of solutions of the form

$$
f_{i k}=\sum_{j=0}^{N} \alpha^{j} f_{i k}^{j}
$$

with $\alpha^{0}=1, f_{i k}^{0}$ a particular solution, and the $f_{i k}^{j}(j>0)$ comprising a basis for the solution space. The $\alpha^{j}(j=1 \ldots N)$ are arbitrary and provide the $N$ degrees of freedom of the solution. By further imposing the inequality constraints, Eqn. (70), the $\alpha^{j}$ values may be restricted to those that give feasible volume fractions. Uniqueness of solutions is not guaranteed and indeed is unlikely in practical examples. Note that additional closure conditions on the relative rotations of the grains have been neglected: on making a complete circuit of the central junction of grains, the final grain must meet the initial grain at the fixed initial boundary. This condition is likely to be of importance in fully three-dimensional examples such as films with grain diameter less than the film thickness, or bulk polycrystals. In a thin, unconstrained lamina, small mismatches may be accommodated by out-of-plane bending at relatively low energetic cost. 
For computation it is convenient to prepare the linear algebra problem in the form

$$
\mathbf{L f}=\mathbf{k} ; \quad \mathbf{f} \geq 0
$$

where $\mathbf{L}$ is the $2 N \times m N$ matrix of coefficients

$$
\left[\begin{array}{ccccccccc}
l_{121} & \cdots & l_{12 m} & -l_{211} & \cdots & -l_{21 m} & 0 & \cdots & 0 \\
0 & \cdots & 0 & l_{231} & \cdots & l_{23 m} & -l_{321} & \cdots & 0 \\
\vdots & & & \ddots & & & & & \\
-l_{1 N 1} & \cdots & -l_{1 N m} & & & & & & l_{N 1 m} \\
1 & \cdots & 1 & 0 & \cdots & 0 & & \cdots & 0 \\
0 & \cdots & 0 & 1 & \cdots & 1 & & \cdots & 0 \\
\vdots & & & \ddots & & & & & \vdots \\
0 & & & & & & & \cdots & 1
\end{array}\right]
$$

while $\mathbf{f}$ and $\mathbf{k}$ are column matrices of length $m N$ and $2 N$ respectively, given by

$$
\mathbf{f}=\left[\begin{array}{c}
f_{11} \\
f_{12} \\
\vdots \\
f_{1 m} \\
f_{21} \\
\vdots \\
f_{N m}
\end{array}\right], \quad \mathbf{k}=\left[\begin{array}{c}
0 \\
\vdots \\
0 \\
1 \\
\vdots \\
1
\end{array}\right]
$$

The first $N$ rows of Eqn. (72) provide the compatibility equations, while rows $N+1 \cdots 2 N$ ensure that the volume fractions sum to unity in each grain. Inverting this system of equations, including the inequalities in (72) is readily achieved using active set methods.

Calculation of example cases indicates that, for a single junction of grains, and a ferroic material with more than two variants, it is normally possible to find a stress-free state. Extending this to a polycrystal with $J$ junctions, each surrounded by, on average, $N$ grains, and with $G$ grains in total, the linear equations number $J N+G$ for the $m G$ unknown volume fractions. In a large region of polycrystalline lamina, let the average number of sides on each grain be $s$ such that $J \sim G s / N$ and $N \sim 2 s /(s-2)$ on average. Then there are approximately $(s+1) / m$ equations per unknown. For realistic values of $s$ and tetragonal martensite, $m=3$, the problem is overdetermined. Thus stress-free states in which the microstructure is uniform within each grain 
are unlikely. The situation is worse in bulk polycrystals, where grains may typically have 14 neighbours. An energy minimizing solution will then involve more complicated microstructure, wherein grains break up into regions with differing mixtures of crystal variants and hence different average states of spontaneous strain. It is commonly observed that grains in ferroic polycrystals show such microstructure, with several sub-grain regions in which bundles of domains form laminated patterns.

\section{Conclusion}

This chapter has presented several models of ferroic materials, treating these materials as composites comprising individual ferroic phases or crystal variants. The close analogy between the divergence-free field variables (stress, electric displacement, magnetic induction) and irrotational variables (strain, electric field, magnetic field) allows several methods from the theory of composites to be generalized easily to multiferroic materials. However, some care is needed in recognizing aspects of the ferroic orders that need distinct modeling approaches. Applications in energy minimizing laminated composites have been described, including the exact evaluation of the moduli of hierarchical laminates, and the compatibility conditions that pertain in crystals and polycrystals of ferroic materials. The methods provide a starting point for the analysis of ferroic composites and their properties.

\section{Acknowledgements}

The author gratefully acknowledges valuable discussions and contributions from Prof. B. Kiefer, Prof. N.T. Tsou, Dr M. Arredondo-Arechavala and K. Holsgrove.

\section{References}

Aizu, K.: Possible species of ferromagnetic, ferroelectric, and ferroelastic crystals. Phys. Rev. B 2, 754-772 (1970). 
Alshits, V. I., Darinskii, A. N., Lothe, J.: On the existence of surface waves in half-infinite anisotropic elastic media with piezoelectric and piezomagnetic properties. Wave Motion 16, 265283 (1992).

Ball, J. M., James, R. D.: Fine phase mixtures as minimizers of energy. Arch. Rational Mech. Anal., 11, 13-52 (1987).

Bhattacharya, K.: Microstructure of Martensite, Why it forms and how it gives rise to the shapememory effect. Oxford University Press (2003).

Bhattacharya, K.: Comparison of the geometrically nonlinear and linear theories of martensitic transformation. Continuum Mech. Thermodyn. 5, 205-242 (1993).

Christensen, R. M.: A critical evaluation for a class of micro-mechanics models. J. Mech. Phys. Solids 38, 379-404 (1990).

De Simone, A, James, R. D.: A constrained theory of magnetoelasticity. J. Mech. Phys. Solids 50, 283-320 (2002).

Dunn, M. L. \& Taya, M.: An analysis of piezoelectric composite materials containing ellipsoidal inhomogeneities. Proc. R. Soc. Lond. A 443, 265-287 (1993).

Eerenstein, W., Mathur, N. D., Scott, J. F.: Multiferroic and magnetoelectric materials. Nature 442, 759-765 (2006).

Eshelby, J.D.: The determination of the elastic field of an ellipsoidal inclusion, and related problems. Proc. R. Soc. Lond. A 241, 376-396 (1957).

Hill, R.: A self-consistent mechanics of composite materials. J. Mech. Phys. Solids 13, 213-222 (1965). 
Huang, J. H., Chiu, Y.-H., Liu, H.-K.: Magneto-electro-elastic Eshelby tensors for a piezoelectric -piezomagnetic composite reinforced by ellipsoidal inclusions. J. Appl. Phys. 83, 5364-5370 (1998).

Huang, J. H., Kuo, W.-S.: The analysis of piezoelectric/piezomagnetic composite materials containing ellipsoidal inclusions. J. Appl. Phys. 81, 1378-1386 (1997).

James, R. D., Hane, K. F.: Martensitic transformations and shape-memory materials. Acta mater. 48, 197-222 (2000).

Kessler, H., Balke, H.: On the local and average energy release in polarization switching phenomena. J. Mech. Phys. Solids 49, 953 - 978 (2001).

Kiefer, B., Lagoudas, D. C.: Magnetic field-induced martensitic variant reorientation in magnetic shape memory alloys. Phil. Mag. 85, 4289-4329 (2005).

Lee, J., Boyd, J. G., Lagoudas, D. C.: Effective properties of three-phase electro-magneto-elastic composites. Int. J. Eng. Sci. 43, 790-825 (2005).

Li, J. Y.: Magnetoelectroelastic multi-inclusion and inhomogeneity problems and their applications in composite materials. Int. J. Eng. Sci. 38, 1993-2011 (2000).

Li, J, Y., Dunn, M. L.: Anisotropic coupled-field inclusion and inhomogeneity problems, Phil. Mag. A, 77, 1341-1350 (1998).

Li, J. Y., Liu, D.: On ferroelectric crystals with engineered domain configurations. J. Mech. Phys. Solids 52, 1719-1742 (2004).

Li, J. Y., Ma, Y. F.: Magnetoelastic modeling of magnetization rotation and variant rearrangement in ferromagnetic shape memory alloys. Mech. Mat. 40, 1022-1036 (2008). 
Mori, T., Tanaka, K.: Average stress in matrix and average elastic energy of materials with misfitting inclusions. Acta. Metal. 21, 571-574 (1973).

Nan, C.-W., Bichurin, M. I., Dong, S., Viehland, D., Srinivasan, G.: Multiferroic magnetoelectric composites: Historical perspective, status, and future directions. J. Appl. Phys. 103, 031101 (2008).

Pons, J., Chernenko, V. A., Santamarta, R., Cesari, E.: Crystal structure of martensitic phases in Ni-Mn-Ga shape memory alloys. Acta Mater. 48, 3027-3038 (2000).

Schmid, H.: Multi-ferroic magnetoelectrics. Ferroelectrics 162, 317-338 (1994).

Shu, Y. C.: Heterogeneous Thin Films of Martensitic Materials. Arch. Rational Mech. Anal. 153, 39-90 (2000).

Shu, Y. C., Bhattacharya, K.: Domain patterns and macroscopic behaviour of ferroelectric materials. Phil. Mag. B, 81, 2021-2054 (2001).

Soh, A. K., Liu, J. X.: On the Constitutive Equations of Magnetoelectroelastic Solids. J. Intell. Mater. Syst. Struct. 16, 597-602 (2005).

Tagantsev, A. K., Cross, L. E., Fousek, J.: Domains in Ferroic Crystals and Thin Films. Springer, New York (2010).

Tsou, N. T., Huber, J. E.: Compatible domain structures and the poling of single crystal ferroelectrics. Mech. Mat. 42, 740-753 (2010).

Tsou, N. T., Huber, J. E., Cocks, A. C. F.: Evolution of compatible laminate domain structures in ferroelectric single crystals. Acta Mater. 61, 670-682 (2013). 
Van Aken, B. B, Rivera, J.-P., Schmid, H., Fiebig, M.: Observation of ferrotoroidic domains. Nature 449, 702-705 (2007).

Wang, B: Three-dimensional analysis of an ellipsoidal inclusion in a piezoelectric material. Int. J. Solids Struct. 29, 293-308 (1992).

Webster, P. J., Ziebeck, K. R. A., Town, S. L., Peak, M. S.: Magnetic order and phase transformation in $\mathrm{Ni}_{2} \mathrm{MnGa}$. Phil. Mag. B, 49, 295-310 (1984).

Weng, G. J.: The theoretical connection between Mori-Tanaka's theory and the HashinShtrikman-Walpole bounds. Int. J. Eng. Sci. 28, 1111-1120 (1990).

Weng, G. J., Wong, D. T.: Thermodynamic driving force in ferroelectric crystals with a rank-2 laminated domain pattern, and a study of enhanced electrostriction. J. Mech. Phys. Solids 57, 571-597 (2009). 